\title{
Advances of genomic science and systems biology in renal transplantation: a review
}

\author{
David Perkins • Meenakshi Verma $\cdot$ Ken J. Park
}

Received: 3 December 2010 /Accepted: 7 January 2011 /Published online: 14 February 2011

(C) The Author(s) 2011. This article is published with open access at Springerlink.com

\begin{abstract}
The diagnosis of rejection in kidney transplant patients is based on histologic classification of a graft biopsy. The current "gold standard" is the Banff 97 criteria; however, there are several limitations in classifying rejection based on biopsy samples. First, a biopsy involves an invasive procedure. Second, there is significant variance among blinded pathologists in the interpretation of a biopsy. And third, there is also variance between the histology and the molecular profiles of a biopsy. To increase the positive predictive value of classifiers of rejection, a Banff committee is developing criteria that integrate histologic and molecular data into a unified classifier that could diagnose and prognose rejection. To develop the most appropriate molecular criteria, there have been studies by multiple groups applying omics technologies in attempts to identify biomarkers of rejection. In this review, we discuss studies using genome-wide data sets of the transcriptome and proteome to investigate acute rejection, chronic allograft dysfunction, and tolerance. We also discuss studies which focus on genetic biomarkers in urine and peripheral blood, which will provide clinicians with minimally invasive methods for monitoring transplant patients. We also discuss emerging technologies, including whole-exome sequencing and RNA-Seq and new bioinformatic and systems biology approaches, which should increase the ability to develop both biomarkers and mechanistic understanding of the rejection process.
\end{abstract}

This article is published as part of the Special Issue on Transplantation and Tolerance

D. Perkins $(\bowtie) \cdot M$. Verma $\cdot$ K. J. Park

Division of Nephrology, Department of Medicine,

University of California San Diego,

San Diego, CA, USA

e-mail: davperkins@ucsd.edu
Keywords Kidney transplantation - Acute rejection . Chronic rejection · Rna-seq · Genomics · Microarrays · Modulated genes

A major challenge in clinical transplantation is to improve long-term graft survival. In kidney transplantation, the development of new immunosuppressive agents has markedly reduced the incidence of acute rejection (AR); however, longterm graft survival has not markedly improved. Because acute rejection is a major risk factor for the development of chronic allograft nephropathy leading to graft loss, it was anticipated that reducing acute rejection would lead to prolonged graft survival. However, decreases in the incidence of AR have not translated into improved long-term outcomes. The biological mechanisms underlying the lack of correlation between reduced rates of AR and graft survival are not currently understood. Are the acute and chronic rejection processes qualitatively different? Are the current immunosuppressive agents designed and selected to preferentially block the AR process? What is the relative contribution of immune/ inflammatory versus nonimmune responses to chronic graft loss? And how can we induce and monitor immune tolerance? Understanding the answer to these questions is essential for the development of biomarkers to diagnose and prognose graft outcomes and also to design novel therapeutic strategies to improve long-term graft survival. This review will focus on recent efforts to apply new advances in genomic science and systems biology to the investigation of these challenges.

The diagnosis of acute renal allograft rejection is commonly prompted by an increase in serum creatinine $(\mathrm{Cr})$ indicating renal injury. However, it is well understood that this is a late marker of rejection only detectable after substantial injury to the graft. Thus, although $\mathrm{Cr}$ can be monitored by minimally invasive methods from serum, it is 
a poor biomarker for early rejection. Following an increase in $\mathrm{Cr}$, AR is confirmed by histological analysis of a graft biopsy based on Banff 97 classification, which is described in the Transplant Pathology Internet Services (http://tpis. upmc.com/TPIShome/changeBody.jsp?url=/tpis/schema/ KNCode95.jsp),[1, 2] Graft biopsy requires an invasive procedure, but is considered the "gold standard" for clinical diagnosis. However, biopsies have several important limitations [1-5]. First, the tubulitis and arteritis lesions of AR are often focal creating the potential for underdiagnosis [5]. Second, rejection may be a continuum rather than discrete categories or levels; for example, "borderline" rejection lack criteria for AR but show progressive increase in $\mathrm{Cr}$ over time [6]. Third, histological scoring does not incorporate the underlying molecular parameters, which require other approaches, including omics technologies, for assessment [7]. Fourth, agreement among multiple observers has shown variability, particularly in evaluation of borderline lesions and chronic allograft nephropathy. For example, one study reported disagreement in $26 \%, 57 \%$, and $22 \%$ of cases assigning a diagnosis of AR, borderline rejection, and no AR, respectively [4]. Thus, the Banff 97 classification has provided a valuable set of criteria to diagnose biopsies and to evaluate and compare clinical studies; however, the lack of complete interobserver reproducibility makes the gold standard far from perfect. To address this challenge, current efforts are underway to combine both histological and omics data into a unified diagnostic criteria [8].

\section{Genomics}

The human genome project began in 1990 under the guidance and combined effort of NIH and US Department of Energy. The generation of a draft genome in 2001 created a great deal of excitement and interest in the field of genomics and illustrated its potential for application to disease states, including transplantation [9, 10]. The availability of the sequence of the human genome facilitated technological advancements, including analysis of the transcriptome, proteome, and genome (SNPs), which are collectively termed as "omics". The bulk of investigations in omics in transplantation have been based on microarray studies of the transcriptome which are discussed in this review. In addition, we discuss several studies analyzing the proteome.

\section{Molecular markers in acute rejection}

The Organ Procurement and Transplant Network (OPTN) database indicates that the number of kidney transplants in the last decade has increased by $31 \%$. The current survival rate is $84 \%$ and $64 \%$ for 5 and 10 years, respectively. Thus, despite the advances in immunosupression, we continue to observe ARs in approximately $11 \%$ [11] of patients in the post-transplant period and high rates of long-term graft failure. As discussed, the Banff classification for renal allograft pathology is the current gold standard for diagnosing $\mathrm{AR}$ and frequently influences therapeutic decisions. Several groups have applied microarray technology to investigate AR. Current versions of microarrays can evaluate more than 55,000 probes $[12,13]$. Currently, there are several commercially available microarray platforms, including Affymetrix and Illumina. Affymetrix uses a solid platform, whereas Illumina uses microscopic beads [12]. The MicroArray Quality Control project evaluated multiple test sites and alternative technology platforms [14]. They demonstrated a high level of intraplatform consistency among different test sites and interplatform concordance in the identification of differentially expressed genes. Nevertheless, a comparison of results of the analysis of kidney biopsy samples among multiple groups reveals low correlation in the gene signature identified in each experiment. This could be due to technical or biological variance, or to different statistical algorithms applied to the data. Recent reviews define standards and ontologies and procedures to normalize and transform microarray data $[15,16]$.

In an early study of renal biopsy samples with microarrays, Sarwal et al. analyzed AR with cDNA spotted arrays [17]. They identified significantly modulated genes that were previously associated with AR, genes with functions not previously known to be involved in AR, as well as ESTs. A limitation of these early studies is that modulated genes were identified only by statistical significance or expression ratios, thus biological interpretations were based on prior knowledge.

In an attempt to evaluate specific biological responses in AR, Halloran and coworkers identified pathogen-based transcripts (PBTs) that were selected based on studies of mouse models of rejection. One study analyzed the response of murine kidneys to recombinant IFN $\gamma$ and to transplant rejection to identify IFN $\gamma$-dependent rejectioninduced transcripts [18]. They observed a robust overlap between transcripts modulated during rejection and the IFN $\gamma$-induced transcripts suggesting a major role for IFN $\gamma$ during rejection. The response was modulated by both host and donor IFN $\gamma$ receptors. Although IFN $\gamma$ is highly upregulated during rejection, it is not essential for rejection in murine models since rejection is not delayed in IFN $\gamma$ knockout strains illustrating the complexity and the multiple mechanisms involved in the rejection process [19]. In an analysis of cytotoxic T cell transcripts, the same group identified modulated transcripts from human cytotoxic $\mathrm{T}$ cells (CATS) and showed that they correlate with the burden of CTL and effector memory T cells in human 
kidney transplant biopsies [20]. Furthermore, the CATS were more accurate than classification based on a single transcript.

Muellar et al. extended these studies in an analysis of six pathogenesis-based transcript sets, which were based on three rejection mechanisms, namely cytotoxic $\mathrm{T}$ cell infiltration, interferon-gamma effects, and parenchymal deterioration [21]. The PBTs were derived from experimental mouse models and were used to study transcriptome disturbances in 143 kidney transplant biopsies. The PBT scores correlated strongly with histopathologic diagnoses of rejection, which had high scores, and biopsies without rejection, which had low scores. They found that the PBT scores correlated well with the Banff classification in most areas, but there were differences noted particularly in the borderline diagnosis. The Banff 97 criteria defines $\mathrm{T}$ cellmediated rejection $1 \mathrm{~A}$ as having moderate tubulitis lesions t2; however, the PBT study found that the molecular scoring did not differentiate biopsies labeled $\mathrm{t} 1$ or $\mathrm{t} 2[2$, 7]. In summary, the molecular markers correlated well with rejection and the authors suggested the use of PBTs as a quantitative measure in the assessment of rejection in renal transplants.

Although these studies discussed above identified genes that were significantly modulated during AR, the correlation between the genes identified in these multiple studies was low. There are several potential explanations for this observation. First, although the data was appropriately analyzed, it is important to consider that approximately 20,000-55,000 features were statistically analyzed in a small number of samples; thus, the statistical power may be inadequate to identify all modulated genes. This may be particularly relevant to genes with low-expression levels that show decreased reproducibility in quality analysis evaluations by several groups. Second, since each study selected a relatively small number of significant genes for final analysis from a large number of modulated genes, subtle changes in the statistical analyses could identify different subsets of modulated genes. Third, it is possible that clinical or experimental variables confound the studies making the application of reproducible research of limited success. A simple solution to this problem would be to increase the number of samples in a study; however, this is limited by the high cost of these experiments. Alternatively, a practical approach is to combine multiple studies in an integrated analysis.

The NIH and most journals require the deposition of microarray data in large online databases, such as the National Center for Biotechnology Information (NCBI) gene expression omnibus (GEO), which is publicly available to scientists and researchers and contains raw microarray expression data. The NCBI reports that the GEO database contains more than a billion gene expression measurements [22]. Availability of such databases provides experimental, control, and comparison groups, which obviously increases the power of integrative data analysis studies.

The integration of data analysis in genetic and genomic studies has many challenges (reviewed in [23]). In contrast to traditional meta-analysis studies, which focus on a single or small number of variables, genomic datasets, including microarray results, have a large number of variables (genes). In a multiplex meta-analysis designed to identify the core immune response genes as defined by the Molecular Signatures Database [24], a comparative analysis of five studies of AR from kidney, lung, and heart transplantation. Their results showed that a parametric method based on a meta effect estimate was superior to a non-parametric rank test in identifying immune response genes in these datasets. This illustrated the importance of the statistical approach applied in a multiplex meta-analysis [25].

Saint-Mezard et al. conducted analyses of the GEO datasets (GSE343, GSE9493, and GSE1563) originally generated by the transplant centers at Stanford, Cleveland Clinic, Hopital Penon in Paris, France. This study identified 70 genes defined as AR transcript set, which were significantly regulated in the three datasets, as well as a non-human primate model of AR [26]. The authors also suggested that the gene sets could be used to assess efficacy of treatment since downregulation of the immune system could be detected prior to the detection of changes on histopathology.

Our group has similarly analyzed the publicly available Alberta dataset (GSE21374) to identify subcategories of AR and used the Paris dataset (GSE9493) as a validation set. Preliminary results identified 983 probe sets, which were significantly different between rejecting and nonrejecting groups (false discovery rate $<0.001$ ). Using clustering algorithms, we identified clusters that segregated the acute and borderline groups. One hundred seventy-one genes were significantly, differentially expressed between the AR and borderline clusters. To investigate the biological functions of the differentially expressed genes, we identified significant Gene Ontology biologic process, which included 16 genes involved in death and 60 genes involved in immune response, including positive regulation of immune system, leukocyte activation, innate immune response, antigen processing and presentation.

Significant KEGG pathways (http://www.genome.jp/ kegg/) included TLR and chemokine signaling, allograft rejection, antigen processing and presentation, NK cell mediated cytotoxicity, graft-versus-host disease, cell adhesion molecules, cytokine-cytokine receptor interaction, FC gamma R-mediated phagocytosis, leukocyte transendothelial migration, and NOD-like receptor signaling. Represen- 
tation of differential expression with heat maps showed higher expression of the 171 genes in cluster 1 compared to cluster 2. Comparison of histologic scores showed increased glomerulitis and interstitial inflammation scores in cluster 1. The correlation coefficient between histologic scores and geometric mean of expression of the 171 genes was significant for glomerulitis, interstitial inflammation, tubulitis, and intimal vasculitis. AUC was greater for cluster 1 (0.93) compared to cluster $2(0.47)$ for predicting AR based on the 171 genes. Using Paris set as an independent data set for validation, rejection samples were also clustered into two groups using these 171 genes. Cluster with higher expression of these 171 genes had greater severity of rejection.

\section{Molecular markers in chronic rejection}

According to the OPTN annual report, the 1-year graft survival for kidney transplants was $91 \%$ at 1 year, but decreases to $43.3 \%$ for deceased donor transplants and $59.3 \%$ for living donor transplants at 10 years. Even though immunosuppression has been successful in decreasing the incidence of AR and increasing 1-year allograft survival rates to $>90 \%$, many grafts develop chronic allograft nephropathy, which often leads to graft failure.

Nakorchevsky et al. analyzed 33 transplant biopsies that were classified into Banff stages 0-3 of Interstitial Fibrosis and Tubular atrophy (IFTA) [27]. They conducted proteogenomic analysis of the samples using shotgun proteomics [27] and grouped the biopsies based on clinical characteristics [27]. They identified 904 differentially expressed proteins and noted that for b0 classification (histologically normal kidney), the proteins were related to normal biologic functions such as aminoacid metabolism [27]. However, for the remaining stages of IFTA, the upregulated proteins were related to cell injury, complement system, fibrosis, and actin cytoskeleton signaling [27]. These results showed that protegenomic analyses can identify differentially expressed proteins and define the mechanistic pathways associated with chronic rejection [27].

Kainz et al. conducted a genome-wide analysis of donor organs before transplantation and at 1 year post-transplant dividing the groups based on good or poor graft function [28]. They identified 52 genes that were differentially expressed between the two groups [28]. The genes that were upregulated in subjects with poor graft function were related to immune system/complement pathway, signal transduction, and oxidative stress [28]. Genes that were upregulated in patients with good graft function were related to normal biologic functions [28].

Scherer et al. analyzed the gene expression profiles of patients at 3 months following transplant who subsequently developed IFTA identified in 6-month protocol biopsies [29]. The comparison group was transplant patients with no evidence of IFTA on 6-month biopsies [29]. They found that upregulated genes in the IFTA group were related to immune sytem, cell signaling, and stress [29]. The second group of patients without IFTA had upregulated genes related to cell function and metabolism [29]. The authors suggested that the molecular signature was present even prior to observation of any histologic changes on graft biopsies [29].

Rodder et al. looked at 29 renal allograft biopsies for cause to assess if matrix mettaloproteinases, belonging to the metazincin family, were differentially expressed in CAN [30]. They detected elevated levels of MMP-7, THBS-2, and other mettaloproteinases related to extracellular remodeling [30].

Kurian et al. also investigated peripheral blood to identify biomarkers of CAN [31]. They found that their accuracy for predicting CAN classes based on gene sets was $80 \%$ for mild CAN and $92 \%$ for moderate to severe CAN [31]. They attempted to reduce the number of biomarkers and showed that with as few as 50 gene sets, they were able to obtain significant identification of CAN [31].

\section{Minimally invasive methods for monitoring}

A major limitation in the analysis of graft biopsy samples by microarray technology is the requirement for an invasive biopsy procedure. Due to the invasive nature of the technique, it is not appropriate for frequent monitoring for AR. Thus, there is considerable interest in developing minimally invasive approaches, for example, monitoring peripheral blood lymphocytes (PBL) to diagnose AR. Flechner et al. analyzed both PBL and kidney biopsy samples in wellfunctioning transplants without rejection, transplants with AR, and kidneys with dysfunction without AR [32]. They identified unique signatures of differentially expressed genes in both the biopsy and PBL samples. Interestingly, the modulated genes in the graft and PBL, although both contained immune genes, were poorly correlated, suggesting that the subsets of cells in the periphery had different states of activation or differentiation than the cells that migrated to the graft. Importantly, this study provided proof of principle that minimally invasive monitoring of PBL could be an effective tool to diagnose AR.

Gunther et al. conducted a closed-cohort case-control study, also analyzing whole blood samples from patients with biopsy-proven rejection who served as cases. Controls were transplant recipients without rejection, patients with borderline rejection, and 20 normal subjects [33]. They discovered 183 probe sets, which generated a refined list of 11 genes, which were differentially expressed in the AR 
group. The probe sets represented three distinct biologic pathways involving immune mechanisms, signal transduction, and cytoskeleton reorganization. They also identified genes related to apoptosis, including ANP32A, a gene which encodes a proapoptoic factor, so not previously associated with AR. The role of specific genes and signatures needs to be evaluated in a larger prospective study.

Peripheral blood monitoring of AR in heart transplant patients has also been evaluated. Horwitz et al. identified a gene expression profile that identified AR from control samples [34]. Interestingly, an analysis of expression profiles following treatment for AR showed an intermediate level of expression for most genes suggesting the persistence of low-level inflammation/immunity. Another study by Deng et al. also identified a panel of modulated genes in PBL that correlates with AR in heart transplant patients [35]. Based on genes selected by the microarray studies plus genes selected based on prior knowledge of immune responses, a commercial PCR-based assay (AlloMap ${ }^{\mathrm{TM}}$ ) was produced and FDA-approved for use in diagnosis of AR in heart transplant patients [36]. Although future refinements in the diagnostic algorithms will be important, these studies demonstrate the feasibility of developing minimally invasive tests for AR.

For kidney transplant patients, an alternative to $\mathrm{PBL}$ is the monitoring of urine samples by PCR. Studies by Suthanthiran and coworkers have identified AR based on granzyme B. Notably granzyme B levels differentiated AR from no rejection, as well as from urinary tract infection. In a subsequent study, they showed that IP-10 and CXCR3 mRNA in urine correlated with AR. Correlations with acute kidney injury or development of chronic allograft nephropathy have not yet been identified.

Since translation of mRNA produces proteins that perform most biological functions, analysis of the differentially expressed proteins offers an alternative approach for the diagnosis of AR. Sarwal et al. analyzed biomarkers in 92 samples of urine from kidney transplant patients with AR, stable grafts, proteinuria, and healthy controls [37]. They identified 1,446 urinary proteins, plus additional proteins that correlate with each group. They identified nine urine proteins unique to patients with $\mathrm{AR}$, and demonstrated reduced levels of uromodulin in patients with rejecting grafts. The analysis of the proteome remains technically more challenging than microarray studies, but offers increased insight into putative biological functions during AR.

\section{Molecular markers of tolerance}

Biomarkers that diagnose or predict AR would provide an important clinical tool for transplant physicians; however, to effectively select immunosuppressive agents and adjust dosages, it will also be important to develop biomarkers to assess immune tolerance. For this discussion, we will consider operational tolerance as the absence of graft rejection in the absence of immunosuppressive therapy. However, it is important to remember that tolerance is complex and likely involves multiple mechanisms, including regulation (by Treg cells), anergy, clonal deletion, as well as suppression. Thus, it will also be important for future studies to delineate the biological mechanisms underlying "operational tolerance" in transplantation.

Brouard et al. analyzed kidney transplant patients with stable renal function and taking no immunosuppressive medications by microarray analysis of peripheral blood samples [38]. The study was limited to 17 tolerant patients due to the scarcity of tolerant patients, but included groups with AR, chronic rejection, minimal immunosuppressive therapy with steroid monotherapy, stable, and normal. The tolerant patients were divided into training $(n=5)$ and test $(n=12)$ sets. Analysis identified a minimal set of 49 genes that were significantly, differentially expressed in the drugfree operationally tolerant group. The gene signature represents reduced costimulatory signaling, immune quiescence, apoptosis, and memory $\mathrm{T}$ cell responses, which are consistent with our understanding of tolerance from experimental models. Interestingly, although TGF $\beta$ was not modulated, $27 \%$ of the genes in the tolerance footprint are regulated by TGF $\beta$. Due to the small number of tolerant subjects, it will be crucial to confirm these intriguing results in a prospective study, perhaps by withdrawing immunosuppression in stable patients with the tolerant footprint.

It has been observed that liver transplant patients have fewer episodes of AR and operational tolerance is more common, occurring in approximately $20 \%$ of transplant recipients. Martinez-Llordella et al. analyzed multiple parameters, including gene expression with microarrays in peripheral blood of 16 tolerant liver transplant patients and identified a signature of "operational tolerance." Signature genes included $\mathrm{T}$ cell receptor and NK receptors. Interestingly, the significantly modulated genes were poorly correlated with the tolerance signature in PBL in kidney transplant patients. These differences may be due to the differences between kidney and liver transplantation or due to technical variation in the small sample size, in which only a small proportion of the tolerant profile are statistically identified.

In an analysis of immunosuppressive drug-free operational immune tolerance of 80 drug-free patients with stable renal function, Braud et al. identified 343 significantly modulated genes in the tolerant group [39]. Two hundred twenty three of these genes were also differentially expressed compared with normal controls. The most significant functional classifications of the 343 differentially expressed genes by EASE that were upregulated, included response to wounding, immune 
response and defense response, and functions that were downregulated included immune response, defense response, and response to pathogen. The inclusion of immune and defense responses in both the upregulated and downregulated group suggest a complex regulation of the genes comprising these functional classifications.

More recently, Newell et al. found that renal allograft tolerance was strongly associated with a B cell signature through microarray analysis [40], which is reviewed in this edition.

\section{Recent technological advances}

There have been several recent technological advances that should increase our ability to identify biomarkers and biological mechanisms of tolerance and rejection, following transplantation. The analysis of genetic variants in transplantation has focused on the MHC due to the strong effect size of this locus; however, there are likely many additional variants that affect transplant outcomes. Genome-wide association studies using microarrays can monitor approximately one million single nucleotide polymorphisms (SNPs), but despite these powerful approaches, a large proportion of the genetic basis of many complex diseases has proven difficult to identify [41, 42]. There are multiple potential rationales for the unresolved genetic effects including the role of rare versus common alleles, geneenvironment interactions, and gene-gene epistatic interactions. The increasing feasibility of whole genome and whole-exome sequencing [43, 44], due to increased speed of "next generation" sequencers, and decreased cost may provide powerful tools to increase our understanding of the genome in transplant outcomes [45].

A limitation of microarray analysis of the transcriptome is the limited dynamic range of the quantification of expression values, particularly within the lower levels of expression. It is well established that PCR has a greater dynamic range than microarrays. However, the development of the RNA-Seq technology has greatly increased the sensitivity of RNA analysis. This method directly sequences the RNA sample without hybridization and has several advantages compared to microarrays. In addition to being more quantitataive, RNA-Seq identifies novel start and stops sites and splice junctions, as illustrated by a recent analysis of $\mathrm{T}$ cell activation [46]. RNA-Seq is also a powerful technology to investigate expression of microRNAs, which have been recognized as important regulators of mRNA. In addition to advances in sequencing, parallel advances in bioinformatics and systems biology enhance the power to develop biological understanding from the large datasets generated by the genomic technologies.

\section{Biological importance of modulated genes versus signaling pathway versus network module}

With current genomic technologies, it is not difficult to generate gigabytes of data; thus, our challenge is to develop and apply bioinformatic and systems biology tools to extract understanding of the relevant biological functions. The application of biostatistical approaches to identify significantly modulated genes may select genes that are informative biomarkers; however, relevant biological functions are not identified. To increase the harvesting of biological information, one approach has been to link modulated genes into signaling pathways using interaction networks or KEGG pathways [47, 48]. The focus on pathways increases the power of the analysis. Based on analysis of interaction networks, it is apparent that pathways have crosstalk, and consequently, are linked in subnetworks or modules [49]. Based on these considerations, the analysis of pathways or modules often provides enhanced biological information compared to significance tests.

\section{One size fits all versus personalized medicine}

Until recently, clinical management of transplant patients was a "one-size-fits-all" design. However, with the development of new immunosuppressive agents, transplant clinicians have multiple therapeutic options and need to determine the most appropriate for each clinical case. There are many factors that make each patient unique, including genetic polymorphisms in each genome, different primary disease, different treatments, and environmental effects. Our long-term goal is to develop individualized personalized medicine for each transplant patients; however, to implement personalized medicine, we will need to understand the major effects of these factors and develop therapies appropriate for each case. Since we have not yet achieved this level of understanding, our immediate progress should focus on subsets, e.g., subsets of AR. This is both tractable and should yield valuable information that could suggest different therapeutic strategies for individual specific subsets. With the development of whole-genome sequencing, RNA-Seq and improved proteomic technology, all integrated with emerging bioinformatic and systems biology tools in the not-too-distant future, we can envision our goal of personalized medicine.

\section{Integrated analysis of complimentary datasets}

Experimental approaches have traditionally applied a single technology such as a microarray. To increase the power of 
these studies, one approach is to increase the sample size; however, an alternative strategy, which provides enhanced biological insight, is to combine multiple technologies. For example, an ideal study of transplant biology would combine genomics plus transcriptomics plus proteomics plus clinical metadata analyzed by multivariant approaches, and perhaps, machine learning algorithms. With these integrated approaches which are costly but all currently feasible, we should approach our goal of personalized medicine.

Open Access This article is distributed under the terms of the Creative Commons Attribution Noncommercial License which permits any noncommercial use, distribution, and reproduction in any medium, provided the original author(s) and source are credited

\section{References}

1. Solez K (2010) History of the Banff classification of allograft pathology as it approaches its 20th year. Curr Opin Organ Transplant 15:49-51

2. Racusen LC et al (1999) The Banff 97 working classification of renal allograft pathology. Kidney Int 55:713-723

3. Veronese FV et al (2005) Reproducibility of the Banff classification in subclinical kidney transplant rejection. Clin Transplant 19:518-521

4. Gough J et al (2002) Reproducibility of the Banff schema in reporting protocol biopsies of stable renal allografts. Nephrol Dial Transplant 17:1081-1084

5. Howie AJ (2002) Problems with BANFF. Transplantation 73:1383-1384

6. Matoza JR, Danguilan RA, Chicano S (2008) Impact of Banff borderline acute rejection among renal allograft recipients. Transplant Proc 40:2303-2306

7. Solez K et al (2008) Banff 07 classification of renal allograft pathology: updates and future directions. Am J Transplant 8:753-760

8. Sis B et al (2010) Banff '09 meeting report: antibody mediated graft deterioration and implementation of Banff working groups. Am J Trans 10:464-471

9. Venter JC et al (2001) The sequence of the human genome. Science 291:1304-1351

10. Lander ES et al (2001) Initial sequencing and analysis of the human genome. Nature 409:860-921

11. U.S. Renal Data System, USRDS (2010) Annual data report: atlas of chronic kidney disease and end-stage renal disease in the United States, National Institutes of Health, National Institute of Diabetes and Digestive and Kidney Diseases, Bethesda, MD

12. Weintraub LA, Sarwal MM (2006) Microarrays: a monitoring tool for transplant patients? Transpl Int 19:775-788

13. Barnes M, Freudenberg J, Thompson S, Aronow B, Pavlidis P (2005) Experimental comparison and cross-validation of the Affymetrix and Illumina gene expression analysis platforms. Nucleic Acids Res 33:5914-5923

14. Shi L et al (2006) The MicroArray Quality Control (MAQC) project shows inter- and intraplatform reproducibility of gene expression measurements. Nat Biotechnol 24:1151-1161

15. Stoeckert CJ Jr, Causton HC, Ball CA (2002) Microarray databases: standards and ontologies. Nat Genet 32(Suppl):469-473
16. Quackenbush J (2002) Microarray data normalization and transformation. Nat Genet 32(Suppl):496-501

17. Sarwal M et al (2001) Genomic analysis of renal allograft dysfunction using cDNA microarrays. Transplant Proc 33:297298

18. Famulski KS et al (2006) Changes in the transcriptome in allograft rejection: IFN-gamma-induced transcripts in mouse kidney allografts. Am J Transplant 6:1342-1354

19. Batirel HF, Batirel S, Mitchell RN, Swanson SJ (2005) Interferongamma knockout fails to confer protection against obliteration in heterotopic murine tracheal allografts. J Heart Lung Transplant 24:658-664

20. Hidalgo LG et al (2008) The transcriptome of human cytotoxic T cells: measuring the burden of CTL-associated transcripts in human kidney transplants. Am J Transplant 8:637-646

21. Mueller TF et al (2007) Microarray analysis of rejection in human kidney transplants using pathogenesis-based transcript sets. Am J Transplant 7:2712-2722

22. http://www.ncbi.nlm.nih.gov/geo/info/GEOHandoutFinal.pdf. Accesed on November 10, 2010

23. Hamid, JS et al. (2009) Data integration in genetics and genomics: methods and challenges. Hum Genomics Proteomics

24. Subramanian A et al (2005) Gene set enrichment analysis: a knowledge-based approach for interpreting genome-wide expression profiles. Proc Natl Acad Sci USA 102:1554515550

25. Morgan AA, Khatri P, Jones RH, Sarwal MM, Butte AJ (2010) Comparison of multiplex meta analysis techniques for understanding the acute rejection of solid organ transplants. BMC Bioinformatics 11(Suppl 9):S6

26. Saint-Mezard P et al (2009) Analysis of independent microarray datasets of renal biopsies identifies a robust transcript signature of acute allograft rejection. Transpl Int 22:293-302

27. Nakorchevsky A et al (2010) Molecular mechanisms of chronic kidney transplant rejection via large-scale proteogenomic analysis of tissue biopsies. J Am Soc Nephrol 21:362-373

28. Kainz A et al (2007) Gene-expression profiles and age of donor kidney biopsies obtained before transplantation distinguish medium term graft function. Transplantation 83:1048-1054

29. Scherer A et al (2009) Transcriptome changes in renal allograft protocol biopsies at 3 months precede the onset of interstitial fibrosis/tubular atrophy (IFTA) at 6 months. Nephrol Dial Transplant 24:2567-2575

30. Rodder S et al (2009) Renal allografts with IFTA display distinct expression profiles of metzincins and related genes. Am J Transplant 9:517-526

31. Kurian SM et al (2009) Biomarkers for early and late stage chronic allograft nephropathy by proteogenomic profiling of peripheral blood. PLoS ONE 4:e6212

32. Flechner SM et al (2004) Kidney transplant rejection and tissue injury by gene profiling of biopsies and peripheral blood lymphocytes. Am J Transplant 4:1475-1489

33. Gunther OP et al (2009) Functional genomics analysis of peripheral blood during early acute renal allograft rejection. Transplantation 88:942-951

34. Horwitz PA et al (2004) Detection of cardiac allograft rejection and response to immunosuppressive therapy with peripheral blood gene expression. Circulation 110:3815-3821

35. Deng MC et al (2006) Noninvasive discrimination of rejection in cardiac allograft recipients using gene expression profiling. Am J Transplant 6:150-160

36. Fang KC (2007) Clinical utilities of peripheral blood gene expression profiling in the management of cardiac transplant patients. J Immunotoxicol 4:209-217 
37. Sigdel TK et al (2010) Shotgun proteomics identifies proteins specific for acute renal transplant rejection. Proteomics Clin Appl 4:32-47

38. Brouard S et al (2007) Identification of a peripheral blood transcriptional biomarker panel associated with operational renal allograft tolerance. Proc Natl Acad Sci USA 104:1544815453

39. Braud $\mathrm{C}$ et al (2008) Immunosuppressive drug-free operational immune tolerance in human kidney transplant recipients: Part I. Blood gene expression statistical analysis. J Cell Biochem 103:1681-1692

40. Newell KA et al (2010) Identification of a B cell signature associated with renal transplant tolerance in humans. J Clin Invest 120:1836-1847

41. Schork NJ, Murray SS, Frazer KA, Topol EJ (2009) Common vs. rare allele hypotheses for complex diseases. Curr Opin Genet Dev 19:212-219

42. Frazer KA, Murray SS, Schork NJ, Topol EJ (2009) Human genetic variation and its contribution to complex traits. Nat Rev Genet 10:241-251
43. Cirulli ET et al (2010) Screening the human exome: a comparison of whole genome and whole transcriptome sequencing. Genome Biol 11:R57

44. Choi M et al (2009) Genetic diagnosis by whole exome capture and massively parallel DNA sequencing. Proc Natl Acad Sci USA 106:19096-19101

45. Werner $T$ (2010) Next generation sequencing in functional genomics. Brief Bioinform 11:499-511

46. Grigoryev YA et al (2009) Genome-wide analysis of immune activation in human $\mathrm{T}$ and $\mathrm{B}$ cells reveals distinct classes of alternatively spliced genes. PLoS ONE 4:e7906

47. Lee E, Chuang HY, Kim JW, Ideker T, Lee D (2008) Inferring pathway activity toward precise disease classification. PLoS Comput Biol 4:e1000217

48. Beyer A, Bandyopadhyay S, Ideker T (2007) Integrating physical and genetic maps: from genomes to interaction networks. Nat Rev Genet 8:699-710

49. Ogata Y et al (2009) The prediction of local modular structures in a co-expression network based on gene expression datasets. Genome Inform 23:117-127 